\title{
Correlation between endoscopic and histopathological findings in dogs with chronic gastritis
}

\author{
Ekrem Ç. Çolakoğlu ${ }^{1}$, Kazım Börkü ${ }^{1}$, Ali E. Haydardedeoğlu ${ }^{2}$, \\ Hadi Alihosseini ${ }^{3}$, Oytun O. Şenel ${ }^{4}$, Nihat Yumuşak ${ }^{5}$, \\ Doğukan Özen ${ }^{6}$, Bülent Baş ${ }^{7}$, Levent Uğurlu ${ }^{8}$ \\ ${ }^{1}$ Department of Internal Medicine, ${ }^{4}$ Department of Surgery, ${ }^{6}$ Department of Biostatistics, \\ ${ }^{7}$ Department of Microbiology, Faculty of Veterinary Medicine, \\ Ankara University, 06110 Ankara, Turkey \\ ${ }^{2}$ Department of Internal Medicine, Faculty of Veterinary Medicine, Aksaray University, 68100 Aksaray, Turkey \\ ${ }^{3}$ Veterinary Practitioner, 07000 Antalya, Turkey \\ ${ }^{5}$ Department of Pathology, Faculty of Veterinary Medicine, Harran University, 63000 Şanliurfa, Turkey \\ ${ }^{8}$ Veterinary Practitioner, 06110 Ankara, Turkey \\ borkukazim@gmail.com
}

Received: April 10, $2017 \quad$ Accepted: August 25, 2017

\begin{abstract}
Introduction: Chronic gastritis is a common diagnosis in dogs with signs of chronic vomiting. However, there is no data concerning endoscopic and histopathological agreement in dogs with chronic gastritis. Thus, a question should be raised whether taking gastroduodenal biopsies in dogs with chronic gastritis is necessary or not. Consequently, the purpose of the study was to compare the endoscopic and histopathological agreement in dogs with chronic gastritis. Material and Methods: A total of 22 non-pregnant client-owned dogs with the signs of chronic gastritis were enrolled in this prospective study. Procedures including clinical examination, blood analysis, and diagnostic imaging were performed before anaesthesia. Biopsies obtained from gastroduodenal sites were histopathologically evaluated. A total of 110 gastroduodenal samples were examined. Results: Sixtyeight samples had abnormal histopathology and endoscopy while 11 showed normal histopathological and endoscopic evidence. Conclusion: The obtained data demonstrated that it is not necessary to take extra gastroduodenal biopsies in dogs with evidence of endoscopic gastroduodenitis. We also believe that further prospective studies, including cost and time effectiveness and more specific comparison between endoscopic appearance and histopathology, are necessary to make final recommendations regarding the need of using both procedures for definitive diagnosis.
\end{abstract}

Keywords: diagnosis, dogs, endoscopy, gastroenteritis, histopathology.

\section{Introduction}

Chronic gastritis with various aetiology is a common disease of dogs. An underlying cause of chronic gastritis is rarely identified and $35 \%$ of dogs with chronic vomiting have been diagnosed as affected with chronic gastritis (21). Exact diagnosis of chronic gastritis in dogs has been conventionally confirmed with endoscopy and histopathology (21). Although associations between endoscopic and histopathological findings were reported several times in human medicine $(7,20)$, there is no data concerning endoscopic and histopathological agreement in dogs with chronic gastritis. Thus, a question should be raised whether taking gastroduodenal biopsies in dogs with chronic gastritis is necessary or not. Hence the purpose of the current study was to compare the endoscopic and histopathological agreement in dogs with chronic gastritis.

\section{Material and Methods}

Animals. The study was performed on 29 nonpregnant client-owned dogs referred to the Veterinary Teaching Hospital with the signs of chronic gastritis 
including episodic vomiting, anorexia, haematemesis, and weight loss. The signs had been occurring for more than 14 days. All dogs had also undergone antiparasitic therapy and routine vaccination against rabies, distemper, parainfluenza, herpes virus infection, parvoviral enteritis, and leptospirosis. The dogs were fed super-premium commercial dry feed. Some dogs were excluded from the study when the gastroduodenal biopsies could not be performed or when gastric signs were associated with extragastrointestinal system, gastric tumour, or gastric foreign body. Dogs receiving drug therapy or with impaired renal or liver function were also excluded from the study. Based on these exclusion criteria, a final total of 22 dogs (12 males and 10 females) were used in the study. The mean age of the dogs was $4.45 \pm 1.97$ years.

Study design. Routine clinical procedures including clinical examination, blood analyses, and diagnostic imaging (abdominal radiographs and ultrasonography) were performed in all dogs before gastroduodenoscopy. Endoscopy of the upper gastrointestinal tract was performed under general anaesthesia induced with a combination of propofol (Adeka, Turkey) and isoflurane (Adeka, Turkey). All dogs were fasted for at least $12 \mathrm{~h}$ with $3 \mathrm{~h}$ water deprivation prior to the procedure. All endoscopies were performed by the same physician using a video endoscope (Eickemeyer, Germany) with alligator jaws biopsy forceps (2.30 $\mathrm{mm}$ diameter). The stomach and duodenum were systematically examined in each dog. Biopsy samples were obtained from gastric fundus, body (greater curvature), antrum (including incisura), pylorus, and duodenum. At least three biopsy samples were taken from each site and gently removed from the forceps using a $25 \mathrm{G}$ needle.

Laboratory examinations. Samples obtained from gastroduodenal sites were fixed in $10 \%$ neutralbuffered formalin solution for three days. Then the tissues were washed in running tap water and dehydrated with ethanol $(50 \%, 75 \%, 96 \%$, and $100 \%)$. After the dehydration process, the specimens were placed in xylene and then embedded in paraffin. Sections of $5 \mu \mathrm{m}$ thickness were cut (Leica RM 2125 RT microtome, USA) and stained with haematoxylineosin (HE). All slides were examined under a light microscope (Olympus BX53, Japan) by a blinded pathologist. Morphological and inflammatory features of the biopsy samples (Table 1) were evaluated according to the WSAVA guidelines $(4,22)$. After endoscopy, clinician reported the gastroduodenal endoscopic findings (erosion, ulcer, hypaeremia, granularity, friability, lacteal dilatation) according to the WSAVA guidelines (22).

Rapid urease test to detect the presence of urease activity was performed. Biopsy samples were placed in an urea broth (Oxoid, CM0071, UK) with 10\% and $0.001 \%$ urea and phenol red for $30 \mathrm{~min}$ at room temperature. The presence of colour changing from yellow to pink indicated a positive urease activity.
Biopsy samples obtained from gastric sites were cultured on Helicobacter selective medium (Oxoid, CM0311, UK) and incubated for 8-12 days at $37 \mathrm{C}^{\circ}$ with anaerobic conditions.

All dogs were treated with appropriate medications. Written owner consents were also obtained for the purpose of this study.

Statistical analysis. Descriptive statistics were calculated and presented as percentages for each variable. To evaluate the significant changes in proportions of two assessments, McNemar's test was used. For each gastric site, the overall agreement and agreement coefficient were assessed using Gwet's firstorder agreement coefficient (AC1) statistic (10). The level at $\mathrm{P}<0.05$ was considered as significant for statistical evaluation. Statistical analysis was also performed using Stata 12.1 MP4 statistical software.

\section{Results}

Data were collected from 29 dogs including Terrier type $(\mathrm{n}=5)$, German Shepherd $(\mathrm{n}=3)$, Cocker Spaniel $(\mathrm{n}=3)$, Kangal $(\mathrm{n}=3)$, Golden Retriever $(\mathrm{n}=2)$, Rotweiler $(\mathrm{n}=2)$, Boxer $(\mathrm{n}=2)$, and mixed breeds $(n=9)$. Seven dogs were excluded from the study because of concurrent renal and hepatic disease $(n=2)$, previous drug therapy $(n=3)$, and inadequate biopsy samples $(\mathrm{n}=2)$.

The most common initial clinical sign observed in the dogs was episodic vomiting (12 dogs; 54.5\%). Other clinical signs were weight loss (six dogs; $27.3 \%$ ), anorexia (three dogs; 13.6\%), and haematemesis (one dog; $4.5 \%$ ), respectively.

Lymphoplasmacytic gastritis was the most common histopathological lesion (13 dogs). The other common lesions observed were mucosal atrophy/fibrosis (five dogs) (Fig. 1), neutrophilic inflammation (three dogs), and lymphangiectasia (one dog). Helicobacter spp. was also isolated from 14 biopsy samples of the gastric fundus and body. Rapid urease test was also positive in all samples.

Frequently recorded endoscopic appearance of gastroduodenal sites were hyperaemia, erosion (Fig. 2), friability, granularity, and ulcer.

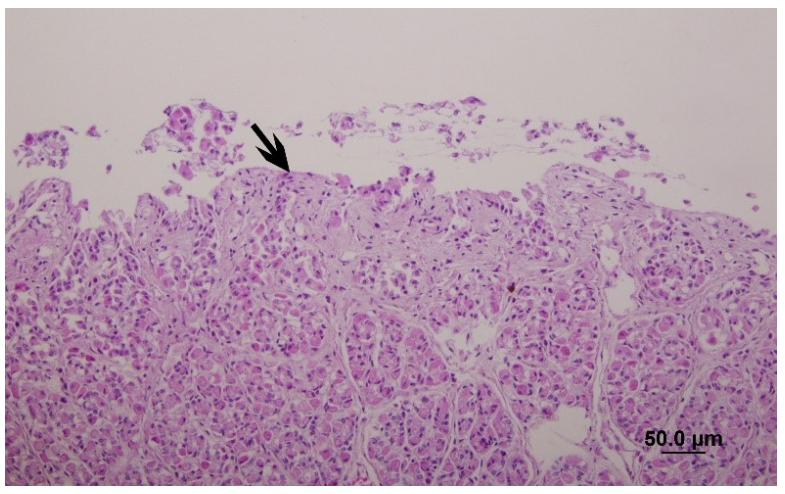

Fig. 1. Atrophy and fibrosis in gastric mucosa (arrow) $200 \times, \mathrm{HE}$ 


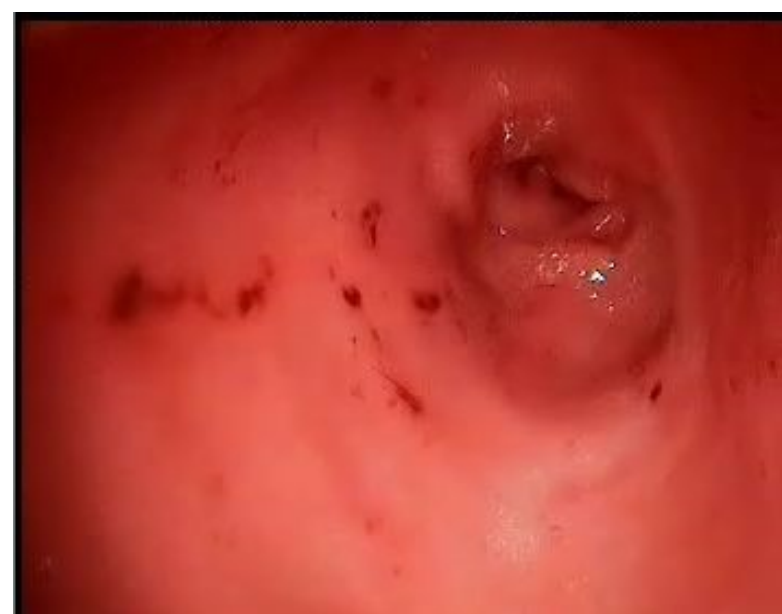

Of the 110 gastroduodenal biopsy samples studied, $68 \quad(86.1 \%)$ samples had abnormal histopathology compared with abnormal endoscopy while $11(13.9 \%)$ showed normal histopathological and endoscopic evidence. The agreement of endoscopy and histopathology results in sites with normal and abnormal endoscopic appearance is shown in Table 2. Of the 37 biopsy samples obtained from endoscopically normal sites, 26 (72.2\%) had abnormal histopathology (Table 2). However, of the 73 biopsy samples obtained from endoscopically abnormal sites, $68(93.2 \%)$ also had abnormal histopathology (Table 2).

Fig. 2. Erosions on gastric antrum

Table 1. Standard form for assessment of the gastric and duodenal mucosa

\begin{tabular}{|c|c|c|c|c|}
\hline Morphological features & Normal & Mild & Moderate & Marked \\
\hline \multicolumn{5}{|l|}{ Surface epithelial injury } \\
\hline \multicolumn{5}{|l|}{ Gastric epithepithelial injury } \\
\hline \multicolumn{5}{|l|}{ Fibrosis/glandular nesting/mucosal atrophy } \\
\hline \multicolumn{5}{|l|}{ Villous stunting (duodenal mucosa) } \\
\hline \multicolumn{5}{|l|}{ Epithelial injury (duodenal mucosa) } \\
\hline \multicolumn{5}{|l|}{ Crypt distension (duodenal mucosa) } \\
\hline \multicolumn{5}{|l|}{ Lacteal dilation (duodenal mucosa) } \\
\hline \multicolumn{5}{|l|}{ Mucosal fibrosis (duodenal mucosa) } \\
\hline Inflammation & Normal & Mild & Moderate & Marked \\
\hline \multicolumn{5}{|l|}{ Intraepithelial lymphocytes } \\
\hline \multicolumn{5}{|l|}{ Lamina propria } \\
\hline \multicolumn{5}{|l|}{ Lamina propria eosinophils } \\
\hline \multicolumn{5}{|l|}{ Lamina propria neutrophils } \\
\hline \multicolumn{5}{|l|}{ Other inflammatory cells } \\
\hline \multicolumn{5}{|l|}{ Gastric lymphofollicular hyperplasia } \\
\hline Final Diagnosis & Normal & Mild & Moderate & Marked \\
\hline \multicolumn{5}{|l|}{ Normal tissue } \\
\hline \multicolumn{5}{|l|}{ Lymphoplasmacytic inflammatory } \\
\hline \multicolumn{5}{|l|}{ Eosinophilic inflammatory } \\
\hline \multicolumn{5}{|l|}{ Neutrophilic inflammatory } \\
\hline \multicolumn{5}{|l|}{ Mucosal atrophy/fibrosis } \\
\hline Lymphangiectasia (duodenal mucosa) & & & & \\
\hline
\end{tabular}

Table 2. Agreement of endoscopic and histopathological lesions in sites with normal and abnormal endoscopic appearance

\begin{tabular}{|c|c|c|c|c|c|c|c|c|}
\hline \multicolumn{9}{|c|}{ Histopathology $\mathrm{y}^{\mathrm{a}}$} \\
\hline \multirow{14}{*}{ 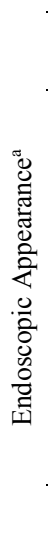 } & & & $\mathrm{Nol}$ & & $\mathrm{Ab}$ & & Agreement & McNemar's \\
\hline & & & $\mathrm{n}$ & $\mathrm{n} \%$ & $\mathrm{n}$ & $\mathrm{n} \%$ & coefficient $^{\mathrm{b}}$ & test $^{\mathrm{c}}$ \\
\hline & \multirow{2}{*}{ Fundus } & Normal & 3 & $30.0 \%$ & 7 & $70.0 \%$ & \multirow{2}{*}{0.358} & \multirow{2}{*}{0.07} \\
\hline & & Abnormal & 1 & $8.3 \%$ & 11 & $91.7 \%$ & & \\
\hline & \multirow{2}{*}{ Body } & Normal & 1 & $33.3 \%$ & 2 & $66.7 \%$ & \multirow{2}{*}{0.829} & \multirow{2}{*}{0.99} \\
\hline & & Abnormal & 1 & $5.3 \%$ & 18 & $94.7 \%$ & & \\
\hline & \multirow{2}{*}{ Antrum } & Normal & 1 & $14.3 \%$ & 6 & $85.7 \%$ & \multirow{2}{*}{0.528} & \multirow{2}{*}{0.125} \\
\hline & & Abnormal & 1 & $6.7 \%$ & 14 & $93.3 \%$ & & \\
\hline & \multirow{2}{*}{ Pylorus } & Normal & 4 & $40.0 \%$ & 6 & $60.0 \%$ & \multirow{2}{*}{0.422} & \multirow{2}{*}{0.125} \\
\hline & & Abnormal & 1 & $8.3 \%$ & 11 & $91.7 \%$ & & \\
\hline & \multirow{2}{*}{ Duodenum } & Normal & 2 & $28.6 \%$ & 5 & $71.4 \%$ & \multirow{2}{*}{0.58} & \multirow{2}{*}{0.219} \\
\hline & & Abnormal & 1 & $6.7 \%$ & 14 & $93.3 \%$ & & \\
\hline & \multirow{2}{*}{ All sites } & Normal & 11 & $29.73 \%$ & 26 & $70.27 \%$ & \multirow{2}{*}{0.556} & \multirow{2}{*}{$<0.001$} \\
\hline & & Abnormal & 5 & $6.85 \%$ & 68 & $93.15 \%$ & & \\
\hline
\end{tabular}

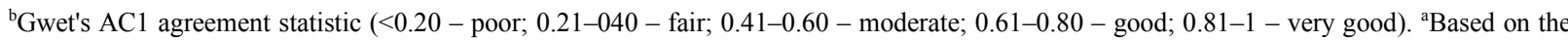
WSAVA guidelines for gastroduodenal histopathology and endoscopy. 'Exact (2-sided) P-values of McNemar's test 


\section{Discussion}

Studies considering agreement of endoscopic and histopathological lesions in patients with gastritis have been published in human medicine $(7,14,17)$. However, clinical usefulness of taking gastroduodenal biopsies in patients with endoscopically-defined gastritis is still unclear (3). Although there are internationally accepted recommendations in collecting gastroduodenal biopsies, cost and time effectiveness is still questionable (3). In veterinary medicine, exact diagnosis of the chronic gastritis in dogs has been confirmed with endoscopy and concurrent biopsy (1). We believe that the study presented here was the first attempt at evaluating the endoscopic and histopathological correlations in dogs with chronic gastritis. The objective of the study was to challenge the hypothesis that there is an agreement between both examinations.

The main cause of the chronic gastritis still remains unknown (9). Many aetiological factors such as allergic, drug or reflux associated, foreign bodyinduced, parasitic, toxic, and uraemic have been reported in dogs with chronic gastritis (5). In this study, many of them have been ruled out by the diagnostic procedures including clinical examination, routine blood analysis, abdominal radiographs, and ultrasonography. Only dogs with the signs of chronic idiopathic gastritis observed for more than 14 days were enrolled in the study as consistent with the reports previously described $(5,16)$. Vomiting, weight loss, and anorexia have been reported as the most common clinical signs in dogs with chronic gastritis $(16,19,21)$, and these were indeed observed in the dogs which were the subjects of the study presented here.

Dogs with chronic gastritis have been histopathologically classified according to predominant cellular infiltrates (21). Lymphoplasmacytic nonulcerative gastritis has been described as the most common form of chronic gastritis in dogs. Eosinophilic, neutrophilic, atrophic, and hypertrophic gastritis are less common $(5,21)$. In the current study, the most common lesion of chronic gastritis was lymphoplasmacytic non-ulcerative gastritis and the other forms were less common. Helicobacter-like organisms have been observed in gastric biopsies of dogs with or without gastritis $(6,18,21)$. Some reports have also indicated the Helicobacters in apparently healthy dogs $(11,13)$. However, isolation of the Helicobacters in apparently healthy dogs causes difficulties in evaluating the relationship between gastritis and helicobacteriosis (12). Although it was not the aim of the study to investigate the association between helicobacteriosis and chronic gastritis in dogs, 14 biopsy samples from the gastric fundus and body revealed the presence of Helicobacter species in bacterial cultures.

Gastroduodenoscopic findings such as ulcer, erosion, hyperaemia, granularity, haemorrhage, and friability of gastroduodenal mucosa have been observed in dogs with endoscopically-defined gastroduodenitis $(12,16)$. In the current study, similar endoscopic findings in dogs were also observed.

The standards of endoscopic and histopathological evaluations in dogs with gastrointestinal disease have been proposed by the World Small Animal Veterinary Association Gastrointestinal Standardization Group (4, 22). Gastrointestinal endoscopy, morphological and inflammatory features of the biopsy samples have also been defined by the same group. The study presented here closely reflects the comparison of the endoscopic and histopathological findings according to the WSAVA guidelines. Although some reports reflecting intestinal and concurrent gastric histopathological abnormalities are available in veterinary medicine (16), no reports related to possible agreement of endoscopy and histopathology in gastroduodenal sites have been published. Data about endoscopic and histopathological correlations have been obtained from the human studies $(4,17,20)$. In a study regarding endoscopic and histopathological agreement in human patients with non-erosive duodenitis (2), $80 \%$ agreement between endoscopic appearance and histopathological abnormalities was observed. In the study of 59 patients with histopathologically-defined gastroduodenitis (8), 25\% of the subjects had normal endoscopy. In another study (1), 38\% of 78 patients with endoscopically-defined gastritis had histopathological inflammation. However, a recent study by Kono et al. (15) aimed to verify the hypothesis of the usefulness of endoscopic atrophy in predicting histological atrophy. This study showed that predicting histopathological atrophy with endoscopic assessment before taking biopsies gives positive results. The current study also showed a moderate to very good agreement between endoscopic and histopathological assessments in gastric body, antrum, pylorus, and duodenum, but a fair agreement in gastric fundus. In a study by Dawson et al. (3), 1,109 patients with endoscopic gastritis (uncomplicated) were retrospectively reviewed. Cost and time effectiveness of taking histopathological biopsies were also investigated. A total of $88.5 \%$ of patients had at least two biopsies taken and no serious histopathological lesions were found in any of the biopsy samples. Despite the recommendations to the contrary, taking histopathological biopsies in patients with uncomplicated gastritis has not been recommended by that study. The question regarding the necessity of taking gastroduodenal biopsies in dogs with chronic idiopathic gastritis still remains to be answered. The data obtained from the results of the current study have shown that it is not necessary to take extra gastroduodenal biopsies in dogs with evidence of endoscopic gastroduodenitis. We also believe that further prospective studies including cost and time 
effectiveness and more specific comparison between endoscopic appearance and histopathology are necessary to make final recommendations regarding the need of using both procedures for definitive diagnosis.

Conflict of Interests Statement: The authors declare that there is no conflict of interests regarding the publication of this article.

Financial Disclosure Statement: This study was supported by grants from Ankara University Scientific Research Council (project no: 10A33380002).

Animal Rights Statement: None required. Written owner consents were obtained.

\section{References}

1. Atkins L., Benedict E.B.: Correlation of gross gastroscopic findings with gastroscopic biopsy in gastritis. N Engl J Med 1956, 254, 641-644.

2. Cotton P.B., Price A.B., Tighe J.R., Beales J.S.M.: Preliminary evaluation of "duodenitis" by endoscopy and biopsy. Br Med J 1973, 3, 430-433.

3. Dawson L., Baker J., Ala K., Shutt J., Jupp J., Bridger S.: Is taking histological biopsies in patients with gastritis a waste of time and money? Gut 2016, 65, 13-14.

4. Day M.J., Bilzer T., Mansell J., Wilcoc B., Hall E.J., Jergens A., Minami T., Willard M., Washabau R.: Histopathological standards for the diagnosis of gastrointestinal inflammation in endoscopic biopsy samples from the dog and cat: a report from the world small animal veterinary association gastrointestinal standardization group. J Comp Path 2008, 138, 1-43.

5. Dunn J.K.: Textbook of small animal medicine. W.B. Saunders, London, 1999.

6. Eaton K.A., Dewhirst F.E., Paster B.J., Tzellas N., Coleman B.E.., Paola J., Sherding R.: Prevalence and varieties of Helicobacter species in dogs from random sources and pet dogs: animal and public health implications. J Clin Microbiol 1996, 34, 3165-3170.

7. Elta G.H., Appelman H.D, Behler E.M., Wilson J.A., Nostrant T.J.: A study of the correlation between endoscopic and histological diagnoses in gastroduodenitis. Am $\mathrm{J}$ Gastroenterol 1987, 82, 749-753.

8. Greenlaw R., Sheahan D.G., Deluca V., Miller D., Myerson D., Myerson P.: Gastroduodenitis. Digestive diseases and sciences $1980,25,660-672$.
9. Guilford W.G.: Idiopathic inflammatory bowel disease. In: Strombeck's Small Animal Gastroenterology, edited by D.R. Strombeck, W.B. Saunders, St. Louis, 1996, pp. 451-486.

10. Gwet K.: Handbook of Inter-Rater Reliability. Publishing Co, Gaithersburg, 2001.

11. Happonen I., Saari S., Castren L., Tyni O., Hänninen M.L., Westermarck E.: Occurrence and topographical mapping of gastric Helicobacter-like organisms and their association with histological changes in apparently healthy dogs and cats. J Vet Med Series A 1996, 43, 305-315.

12. Hermanns W., Kregel K., Breuer W., Lechner J.: Helicobacterlike organisms: histopathological examination of gastric biopsies from dogs and cats. J Comp Pathol 1995, 112, 307-318.

13. Hwang C.Y., Han H.R., Youn H.Y.: Prevelance and clinical characterization of gastric Helicobacter species infection of dogs and cats in Korea. J Vet Sci 2002, 3, 123-133.

14. Ibis M., Arhan M., Odemis B.: The relation between endoscopically diagnosed gastritis and its histologic findings. Turk J Academic Gastroenterol (Akademik Gastroenteroloji) $2009,8,12-17$

15. Kono S., Gotoda T., Yoshida S., Oda I., Kondo H., Gatta L., Naylo G., Dixon M., Moriyasu F., Axon A.: Can endoscopic atrophy predict histological atrophy? Historical study in United Kingdom and Japan. World J Gastroenterol 2015, 21, 1311313123.

16. Lidbury J.A., Suchodolski J.S., Steiner J.M.: Gastric histopathologic abnormalities in dogs: 67 cases (2002-2007). J Am Vet Med Assoc 2009, 234, 1147-1153.

17. Nakagawa S., Kato M., Shimizu Y.: Relationship between histopathologic gastritis and mucosal microvascularity: observations with magnifying endoscopy. Gastrointest Endosc 2003, 58, 71-75.

18. Robić M., Artuković B., Beck A., Gudan A., Svetina A., Grabarević Z.: Histopathological changes in stomachs of dogs with naturally acquired Helicobacter infection. Vet Arhiv 2007, 77, 103-111.

19. Shabestari A.S., Mohammadi M., Jamshidi S., Sasani F., Bahadori A., Oghalaie A.: Assessment of chronic gastritis in pet dogs and its relation with Helicobacter-like organisms. Pak J Biol Sci 2008, 11, 1443-1448.

20. Shanmugasamy K., Bhavani K., Narashiman R., Kotasthane D.S.: Clinical correlation of upper gastrointestinal endoscopic biopsies with histopathological findings and to study the histopathological profile of various neoplastic and non-neoplastic lesions. J Pharm Biomed Sci 2016, 6, 220-224.

21. Simpson K.W.: Gastric disease. In: Textbook of Veterinary Internal Medicine, edited by S.J. Ettinger, E.C. Feldman, W.B. Saunders, St. Louis, 2010, p. 1515.

22. Washabau R.J., Day M.J., Willard M.D., Hall E.J., Jergens A.E., Mansel J., Minami T., Bilzer T.W.: Endoscopic, biopsy, and histopathologic guidelines for the evaluation of gastrointestinal inflammation in companion animals. J Vet Intern Med 2010, 24, $10-26$. 